\title{
Sugarcane Bagasse: A Potential Medium for Fungal Cultures
}

\author{
Arushdeep Sidana and Umar Farooq \\ Molecular and Immuno-Parasitology Laboratory, Department of Microbiology, Faculty of Biotechnology, \\ Shoolini University of Biotechnology and Management Sciences, Solan, Himachal Pradesh 173212, India \\ Correspondence should be addressed to Umar Farooq; ufarooq8@gmail.com
}

Received 19 December 2013; Accepted 18 February 2014; Published 13 March 2014

Academic Editors: X. Geng and R. Su

Copyright ( $\odot 2014$ A. Sidana and U. Farooq. This is an open access article distributed under the Creative Commons Attribution License, which permits unrestricted use, distribution, and reproduction in any medium, provided the original work is properly cited.

\begin{abstract}
Worldwide, sugarcane industries produce tons of sugarcane bagasse as residual/waste material. This residual material is rich in complex lignocellulosic substances and may be used as a low cost carbon and energy source for the growth of fungal species. The present work was aimed at designing a sugarcane waste-based medium as a substitute for expensive commercial media for growing fungal cultures. Eight species of fungi, namely, Aspergillus niger, Candida albicans, Saccharomyces cerevisiae, Fusarium sp., and four unidentified species F1, F2, F3, and F5, were grown on the sugarcane bagasse medium which produced remarkable results and competed with standard media like potato dextrose agar, Sabouraud dextrose agar, and cornmeal agar. The designed medium was able to provide nourishment to the fungi as well as prevent the growth of any bacterial or fungal contaminant. The production of spores was more in the sugarcane medium as compared with standard media. Hence, this study led to the discovery of a new and efficient medium for fungal cultures as well as decrease in the waste disposal expenses and efforts.
\end{abstract}

\section{Introduction}

The cost of all the microbiological media is rising at a fast pace. To tackle this problem some new microbiological media should be designed which are efficient as well as cost effective. This may be achieved by using agricultural wastes as raw materials for microbial media. Utilization of agricultural waste as a substrate for fungal cultures for the production of value added products has been reported which includes cellulase production by some fungi cultured on pineapple waste [1]; carotenoids production is carried out on agricultural waste using Blakeslea trispora [2] and cellulase enzyme production on agricultural waste by Aspergillus niger [3]. Sugarcane bagasse has been also reported as an energy source for the production of lipase by Aspergillus fumigatus [4].

Sugarcane bagasse could be a material of choice for designing a new medium as it is easily available in large volumes. Sugarcane is one of the plants having the highest bioconversion efficiency of capturing sunlight through photosynthesis and is able to fix around 55 tons of dry matter per hectare of land annually. There are thousands of small and large scale industries worldwide which use sugarcane as raw material and produce tons of bagasse every year [5]. The sugarcane stalk consists of two parts, an inner pith containing most of the sucrose and an outer rind with lignocellulosic fibres. During sugar processing, the sugarcane stalk is crushed to extract sucrose [6]. This procedure produces a large volume of residue (approximately $240 \mathrm{~kg}$ of bagasse with $50 \%$ humidity per ton of sugarcane) which contains both crushed rind and pith fibers $[5,7]$. This sugarcane bagasse contains complex lignocellulosic material of which the more abundant component is cellulose (33$36 \%)$, hemicellulose is the second predominant fraction (28$30 \%$ ), and lignin is $22 \%[5,8]$. In spite of the great potential of this residual biomass for the production of fuels, chemicals, and other value added products, the majority of it is burned in sugar mills and alcohol distilleries for energy generation, and a smaller fraction is used for animal feeding, paper, and board making $[9,10]$. Mushroom cultivation on sugarcane bagasse is also practised in some countries. Additionally, with the increase in cane production in response to the growth of global ethanol demand (from 66 to 125 million $\mathrm{m}^{3}$ between 2008 and 2020), more bagasse will be available [11,12]. The use of agriculture waste materials for the production of high value 

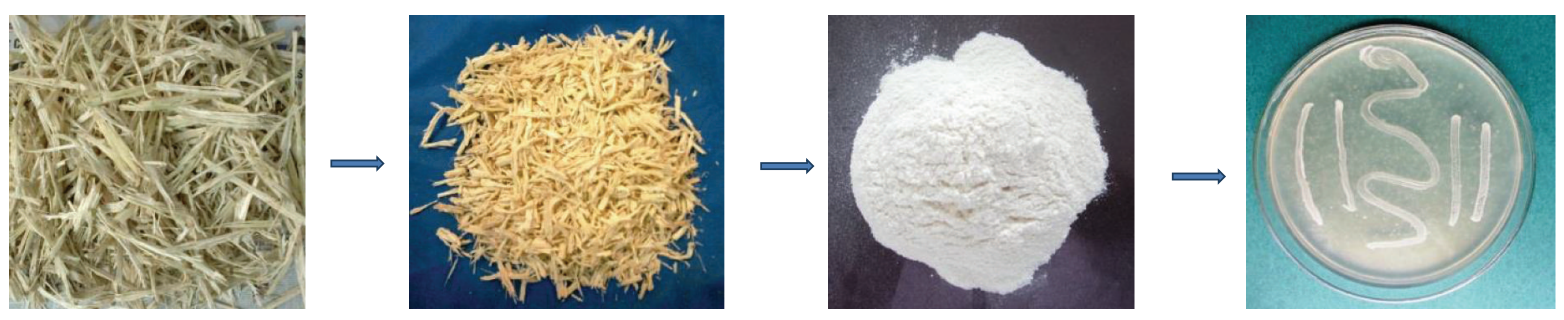

FIgURE 1: Pictorial presentation of the procedure followed for medium designing.

products is also appreciated to decrease the waste disposal efforts and costs.

Generally fungi are grown on potato dextrose agar (PDA), Sabouraud dextrose agar (SDA), or cornmeal agar (CMA) which are very expensive. Basically every fungus requires carbon, nitrogen, and energy source to grow and survive. Sugarcane bagasse may meet these requirements and work as a fungal growth medium and can replace expensive media in the market. This will add a benefit of minimal contamination in the cultures as it does not meet the needs of every microbe. Sugarcane bagasse has been exploited for the production of many high value products but its potential as fungal growth medium has never been reported. The aim of the current study was to design a cost effective and efficient medium for fungal cultures, that is, Aspergillus niger, Candida albicans, Saccharomyces cerevisiae, Fusarium sp., and four unidentified species, that is, F1, F2, F3, and F5, using sugarcane bagasse as raw material.

\section{Materials and Methods}

2.1. Chemicals and Fungal Strains. All the chemicals including sucrose, agar powder, potato dextrose agar, cornmeal agar, and Sabouraud dextrose agar were purchased from Himedia Laboratories, Mumbai, India. Candida albicans and Aspergillus niger were procured from Department of Microbiology, Shoolini Institute of Life Sciences and Business Management, Solan, India; Saccharomyces cerevisiae and Fusarium sp. were procured from Yeast Biology Lab, Shoolini University of Biotechnology and Management Sciences (SUBMS), Solan, India; and F1, F2, F3, and F5 were obtained from Plant Biotechnology Lab, SUBMS, Solan, India, as unidentified fungal growth on plant tissue culture media.

2.2. Designing of Medium. Sugarcane bagasse for medium preparation was procured from local sugarcane juice vendor. The bagasse was then packed in a cloth carry bag to prevent the entry of flies and other insects and dried in shade. After complete drying, the pith portion of the bagasse was extracted manually and the outer rind was discarded. The pith was ground to fine powder in a steel kitchen grinder. The powder thus obtained was strained twice through a muslin cloth. 25 grams of this powder was taken in a $500 \mathrm{~mL}$ conical flask and $400 \mathrm{~mL}$ distilled water was added to it. The flask was placed on a shaker at $150 \mathrm{rpm}$ for 1 week. After a week of shaking the contents of the flask were filtered first through a fine pore muslin cloth and then through Whatman filter paper number 1 . The filtrate $(200 \mathrm{~mL})$ was taken and $8 \mathrm{~g}$ of sucrose powder was added to it. The $\mathrm{pH}$ of the solution was set at 5.6 as that of commercial potato dextrose agar. Four grams of bacteriological agar powder was added to the above solution and dissolved properly by heating. This solution was autoclaved at $121^{\circ} \mathrm{C}$ and 15 psi for $15 \mathrm{~min}$ and solidified after pouring in petri plates for cultivating the eight fungal strains. Each fungus was provided one week of incubation at $25^{\circ} \mathrm{C}$. All the experiments were performed in duplicate. A pictorial representation for the designing of the said medium is presented in Figure 1.

2.3. Comparison with Commercialized Media. For comparison with commercial media all the eight fungal strains were also grown on potato dextrose agar, Sabouraud dextrose agar, and cornmeal agar following conventional procedures. To evaluate the capacity of sugar alone as a fungal growth medium all the eight fungi were also grown on sugar-agardistilled water mixture (lacking sugarcane bagasse powder).

\section{Results and Discussion}

3.1. Preparation of Raw Material. Dried sugarcane bagasse $(500 \mathrm{~g})$, after manual pith extraction, produced 26 grams of fine powder. After shaking and filtration a semiclear whitish liquid was obtained. This liquid on addition of sugar and agar powder was used to grow 8 fungal strains, that is, Aspergillus niger, Fusarium sp., F1, F2, F3, F5, Candida albicans, and Saccharomyces cerevisiae.

\subsection{Fungal Growth on Sugarcane Bagasse Medium (SBM),} Sugar Alone, and Commercial Media. Aspergillus niger had different morphological characteristics in each medium. Maximum growth was obtained in the case of potato dextrose agar and minimum growth was in sugar agar medium (SAM). In case of SBM, visible black coloured spores were more than that in any other media. The colour of colonies was white in SDA, grey in PDA, dusty white in CMA, light grey in SAM, and grey-black in SBM. Maximum vegetative growth was seen in case of PDA and maximum spores were seen in SBM. No bacterial or fungal contamination was there in any of the plates.

Candida albicans has shown similar morphological characteristics in each of the five tested media. Maximum growth was obtained in SDA and least growth was in SAM. SBM exhibited more growth than CMA and SAM, but less than 


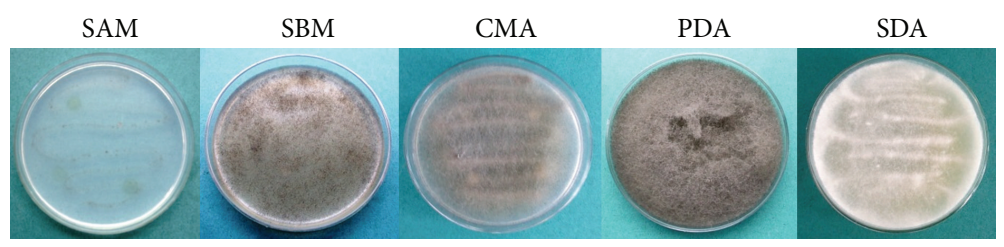

(a)

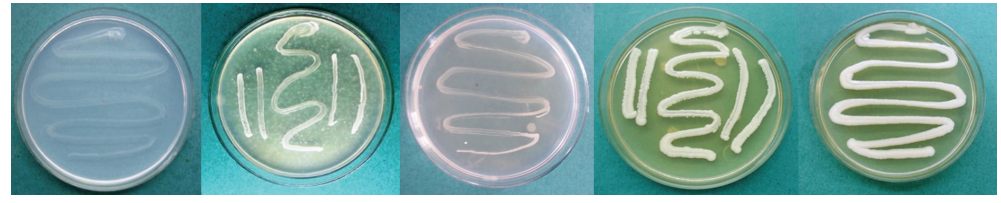

(b)

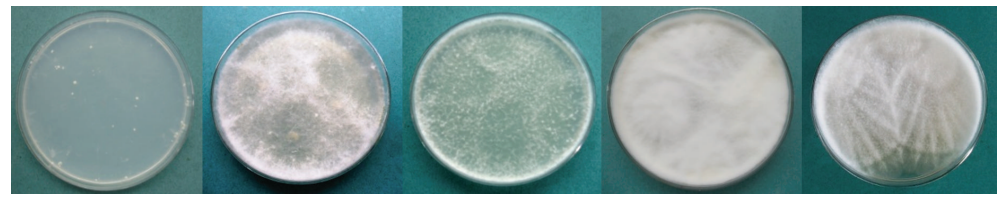

(c)

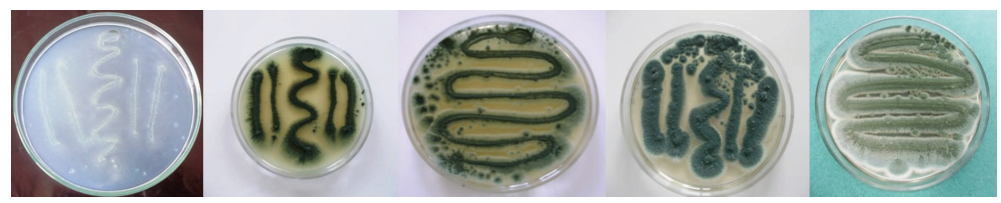

(d)

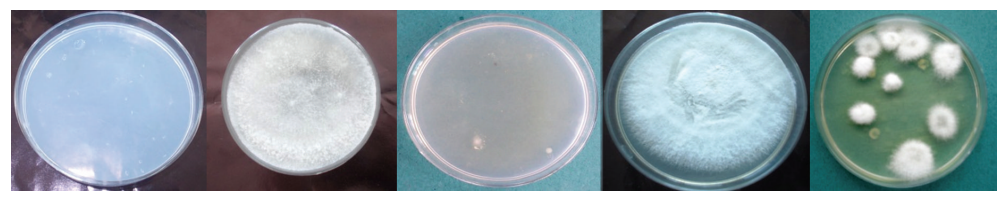

(e)

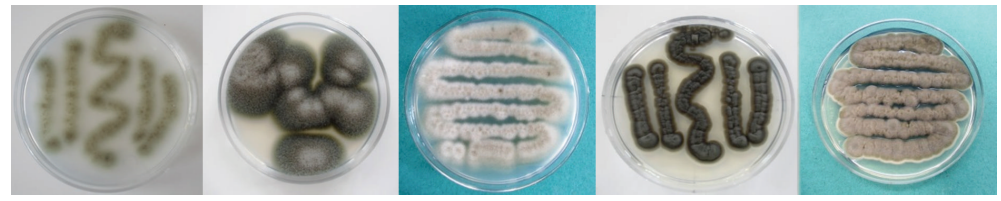

(f)

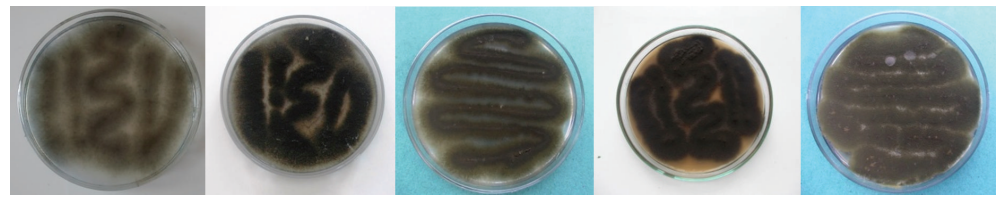

(g)

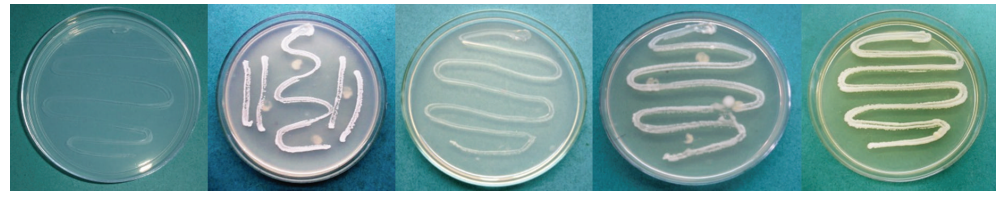

(h)

FIGURE 2: Comparison of growth of different fungal species on SBM, SAM, and three commonly used media in case of (a) Aspergillus niger, (b) Candida albicans, (c) Fusarium sp., (d) F1, (e) F2, (f) F3, (g) F5, and (h) Saccharomyces cerevisiae. 
SDA and PDA. No bacterial or fungal contamination was noticed in any of the media.

F1 has shown different morphological characteristics and growth rates in each of the five media used. Maximum growth was obtained in SDA and minimum growth was in SAM. Growth was similar in the case of SBM and CMA. Most uniform growth was seen in SBM. Fungal colonies on each medium were different in colour and amount of fungal spores.

F2 has shown similar morphological characteristics but different growth rates in all media used. Maximum growth was obtained in SBM and no growth was in the case of SAM and CMA even after seven days of incubation. Maximum vegetative and reproductive structures were obtained in SBM. SDA produced uneven growth.

F3 has shown different morphological characteristics in each of the five media used. Minimum growth was obtained in CMA and SAM; all the other three media have shown similar growth but SBM produced different morphology of the fungal colonies. The purity of the culture grown on SBM was tested by reinoculating the culture in PDA which showed similar morphology as that of the mother culture. Maximum amount of spores was obtained in case of SBM.

F5 has shown similar morphological characteristics and growth rates in each of the five media used except in SAM. Maximum growth was obtained in PDA and minimum was in SAM.

Fusarium sp. has shown similar morphological characteristics but different growth rates in each medium. Maximum growth was obtained in PDA and minimum was in case of CMA and SAM. The growth in SBM and SDA was similar. There were no bacterial or fungal contaminants in any of the plates.

Saccharomyces cerevisiae has shown similar morphological characteristics but different growth rates in each medium. Maximum growth was obtained in SDA and minimum in CMA and SAM. In case of SBM and PDA growth of $S$. cerevisiae was similar to a large extent. No contamination was there in any of the plates. The petri plates showing the growth of different fungi in the developed medium and conventional media are presented in Figure 2.

Sugarcane bagasse contains complex lignocellulosic material which unlike sugar is not charred after autoclaving. This material can be used as low cost energy and carbon source for fungal cultures. Our aim was to carry out a trial to check whether the powdered sugarcane bagasse infusion along with additional sucrose and agar powder is able to nourish fungal cultures or not. For this, eight fungal/yeast species were grown on three conventional and two newly designed media to evaluate the potential of sugarcane bagasse as a potential medium for fungal cultures. Previously reported studies have explained the use of sugarcane bagasse after its pretreatment to yield fermentable sugars for the production of bioethanol [13]. However, in the present study it is used without any pretreatment. The results of this trial were quite satisfactory as the SBM is able to grow some fungi which do not easily grow on conventional media. SBM aids the production of large amount of spores and does not support the growth of any bacterial or fungal contaminants.
Large scale production of sugarcane bagasse dehydrated medium may be carried out by subjecting the solution to spray drying. The study is continued to further improve SBM by adding some more essential nutrients in it to support the growth of more fungal strains.

\section{Conflict of Interests}

The authors declare that there is no conflict of interests regarding the publication of this paper.

\section{Acknowledgments}

The authors thank various laboratories of Shoolini University for providing them with different fungal strains. The authors thanks also go to Shoolini University, Solan, for providing facilities and infrastructure to conduct this study.

\section{References}

[1] P. F. Omojasola, Jilani, P. Omowumi, and S. A. Ibiyemi, "Cellulase production by some fungi cultured on pineapple waste," Nature and Science, vol. 6, no. 2, pp. 64-79, 2008.

[2] E. H. Papaioannou and M. Liakopoulou-Kyriakides, "Agrofood wastes utilization by Blakeslea trispora for carotenoids production," Acta Biochimica Polonica, vol. 59, no. 1, pp. 151-153, 2012.

[3] M. A. Milala, A. Shugaba, A. Gidado, A. C. Ene, and J. A. Wafar, "Studies on the use of agricultural wastes for cellulase enzyme production by Aspegillus niger," Research Journal of Agriculture and Biological Sciences, vol. 1, no. 4, pp. 325-328, 2005.

[4] S. H. A. Naqvi, M. U. Dahot, M. Y. Khan, J. H. Xu, and M. Rafiq, "Usage of sugar cane bagasse as an energy source for the production of lipase by Aspergillus fumigatus," Pakistan Journal of Botany, vol. 45, no. 1, pp. 279-284, 2013.

[5] G. J. V. Betancur and N. Pereira Jr., "Sugar cane bagasse as feedstock for second generation ethanol production. Part I: diluted acid pretreatment optimization," Electronic Journal of Biotechnology, vol. 13, no. 3, pp. 1-9, 2010.

[6] R. Boopathy, "Use of post-harvest sugarcane residue in coastal reclamation: a feasibility study," Sugar Cane International (January/February): 9-13, 2004.

[7] M. O. S. Dias, A. V. Ensinas, S. A. Nebra, R. Maciel Filho, C. E. V. Rossell, and M. R. W. Maciel, "Production of bioethanol and other bio-based materials from sugarcane bagasse: Integration to conventional bioethanol production process," Chemical Engineering Research and Design, vol. 87, no. 9, pp. 1206-1216, 2009.

[8] L. Dawson and R. Boopathy, "Cellulosic ethanol production from sugarcane bagasse without enzymatic saccharification," BioResources, vol. 3, no. 2, pp. 452-460, 2009.

[9] C. E. Wyman, B. E. Dale, R. T. Elander, M. Holtzapple, M. R. Ladisch, and Y. Y. Lee, "Coordinated development of leading biomass pretreatment technologies," Bioresource Technology, vol. 96, no. 18, pp. 1959-1966, 2005.

[10] G. M. Zanin, C. C. Santana, E. P. S. Bon et al., "Brazilian Bioethanol Program," Applied Biochemistry and Biotechnology, vol. 84-86, pp. 1147-1161, 2000.

[11] A. Pandey, C. R. Soccol, P. Nigam, and V. T. Soccol, "Biotechnological potential of agro-industrial residues. I: sugarcane bagasse," Bioresource Technology, vol. 74, no. 1, pp. 69-80, 2000. 
[12] M. Balat and H. Balat, "Recent trends in global production and utilization of bio-ethanol fuel," Applied Energy, vol. 86, no. 11, pp. 2273-2282, 2009.

[13] N. Mosier, C. Wyman, B. Dale et al., "Features of promising technologies for pretreatment of lignocellulosic biomass," Bioresource Technology, vol. 96, no. 6, pp. 673-686, 2005. 

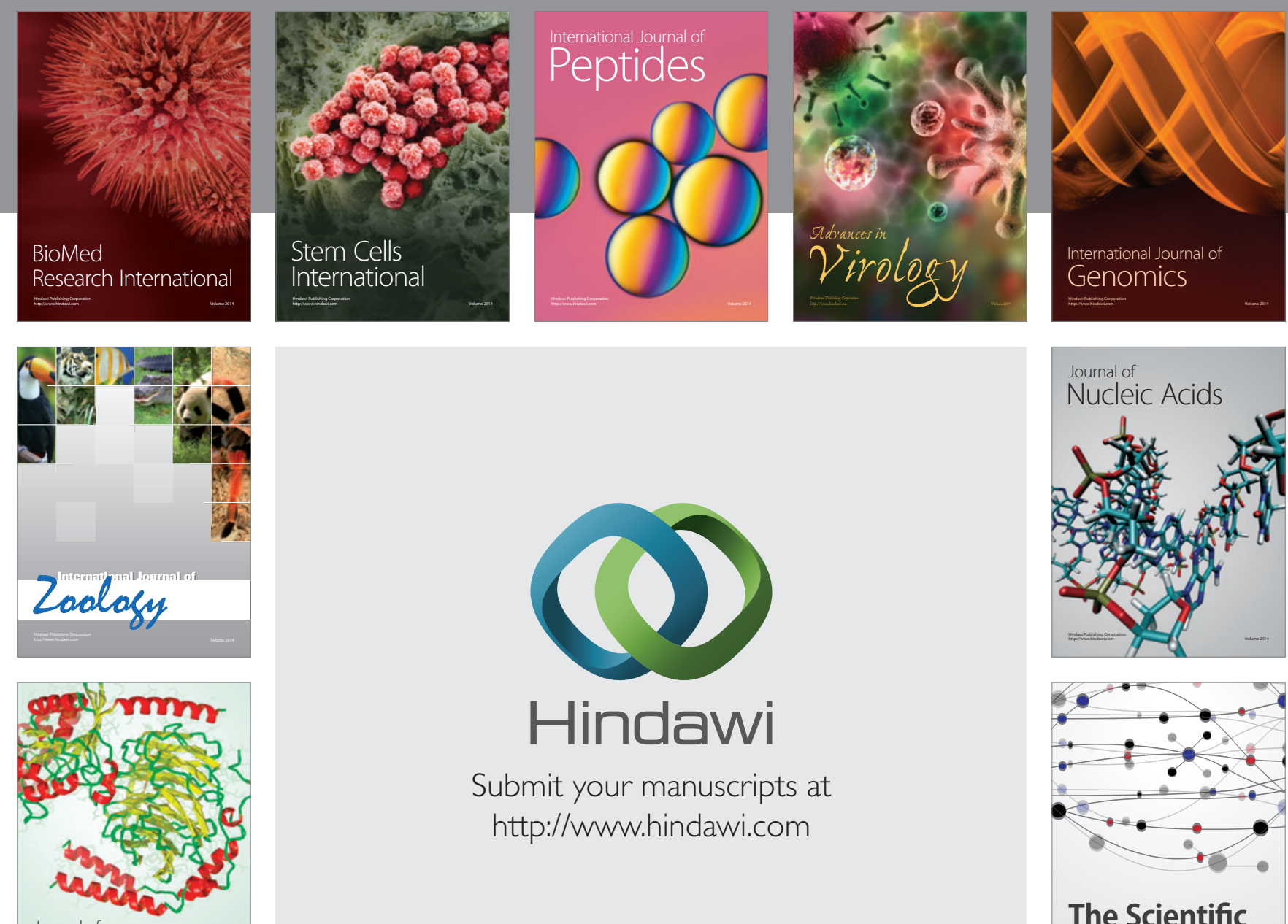

Submit your manuscripts at

http://www.hindawi.com

Journal of
Signal Transduction
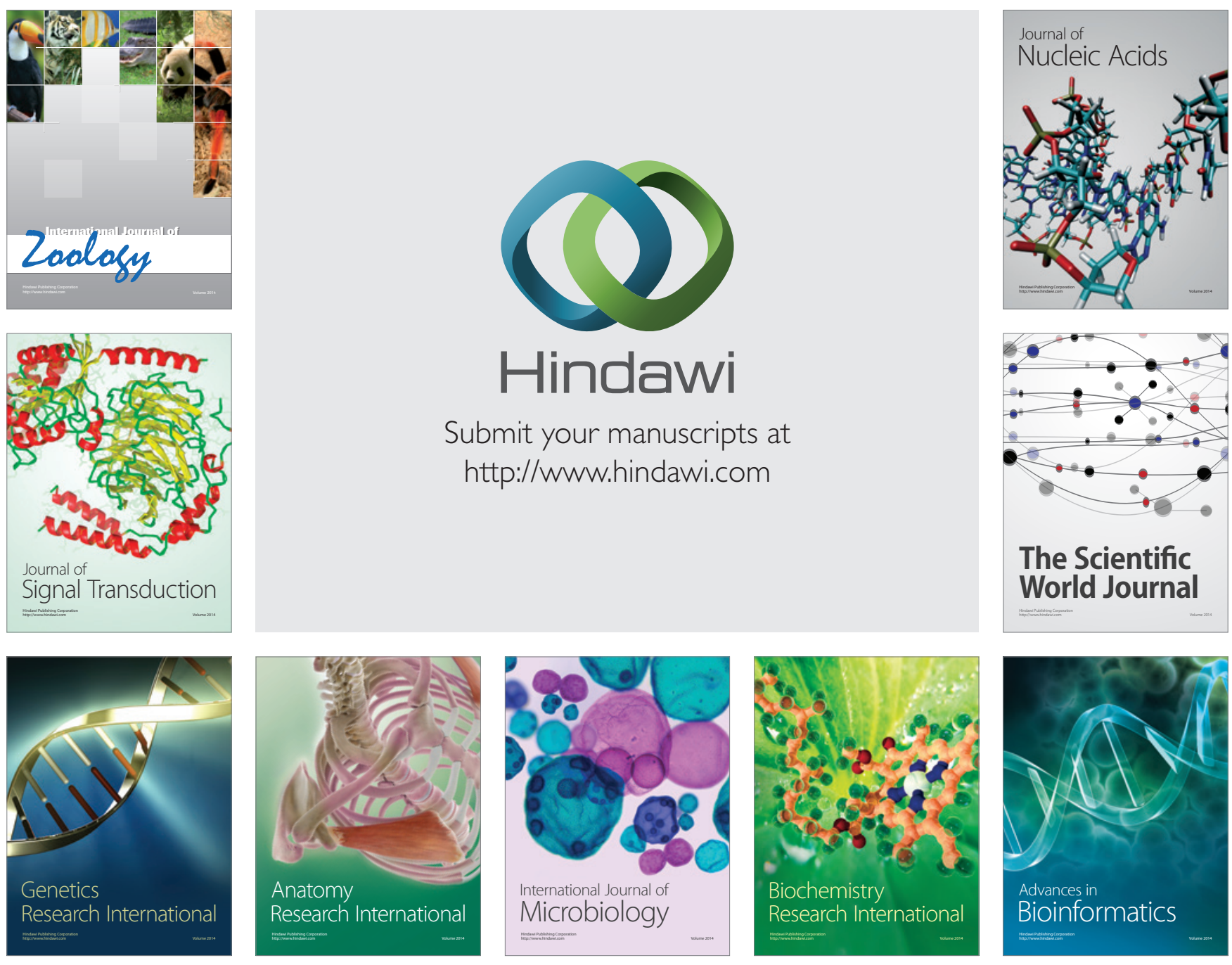

The Scientific World Journal
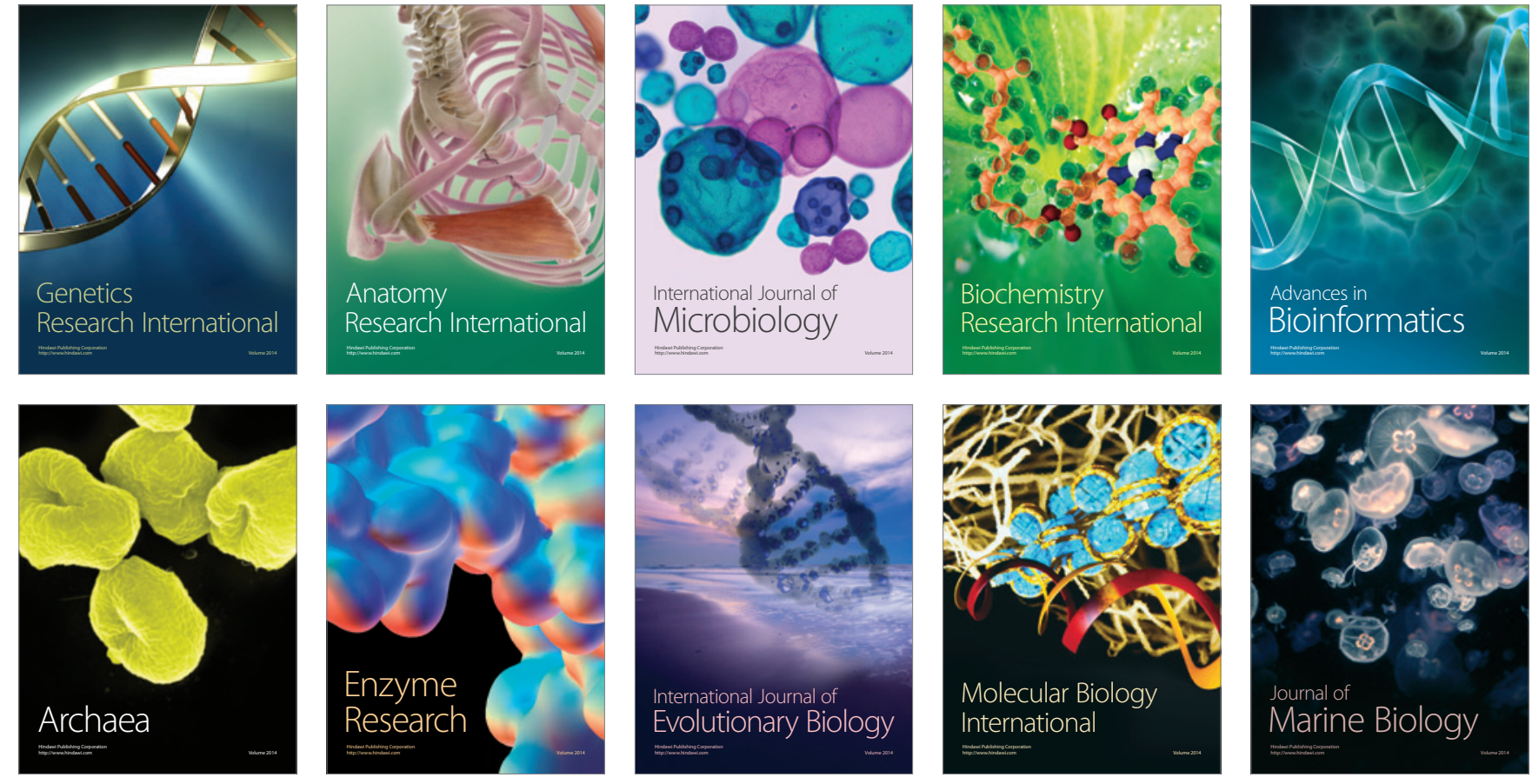